\title{
UN PALAMITE RUSSE DU DÉBUT DU XXÈME SIÈCLE : LE HIÉROMOINE ANTOINE BOULATOVITCH ET SA DOCTRINE SUR L'ÉNERGIE DIVINE ${ }^{1}$
}

\begin{abstract}
Il est difficile de concevoir qu'à l'aube du XX siècle une querelle théologique ait pu ... se propager en Russie parmi les couches d'une société déjà largement sécularisée et provoquer ainsi une crise comparable aux pires excès des disputes christologiques dans l'empire byzantin des $V^{e}-V I^{e}$ siècles. Il y a là matière à paradoxe, ce n'est certainement pas le seul.
\end{abstract}

Antoine Nivière ${ }^{2}$

La pensée théologique du hiéromoine Antoine Boulatovitch (18701919) reste pratiquement inconnue aux patrologues et aux historiens de la philosophie à l'Occident, elle est mal étudiée jusqu'au présent même en Russie, où au début du $X X^{\mathrm{e}}$ siècle avait éclaté la querelle théologique dont le père Antoine est devenu l'un des plus actifs participants. ${ }^{3}$

(1) Les abréviations employées dans cet article :

Ар. - Иеросхимонах Антоний (Булатович), Апология веры во Иля Божие и во Имя Иисус [Hiéromoine Antoine Boulatovitch, L'apologie de la foi en le Nom de Dieu et en le Nom de Jesus] (Москва, 1913).

МР - Ірем, Моя мысль во Христе. О Деятельности (Энергии) Божества [Ma pensée en Christ. Sur l'Activité (l'Énergie) de la Divinité] (Петроград, 1914).

JF - Ірем, Оправдание веры в Непобедимое, Непостижимое, Божественное Имя Господа нашего Иисуса Христа [La justification de la foi en le Nom Invincible, Incompréhensible, Divin de notre Seigneur le Christ Jesus] (Петроград, 1917).

(2) A. Nivière, L'onomatodoxie: une crise religieuse à la veille de la révolution, dans : Mille ans de Christianisme russe: 988-1988 (Paris, 1989) 285.

(3) Sur la discussion entre les onomatodoxes (имяславизь, les glorificateurs du nom) et les onomatoclastes (имябориьь, les ennemis du nom; les onomatodoxes appelaient ainsi leurs adversaires, comme les iconodules appelaient autrefois les iconoclastes) voir: A. Nivière, Le mouvement onomatodoxe: une querelle théologique parmi les moines russes du Mont-Athos (19071914) (PhD diss., Paris, Sorbonne, 1987) ; T. E. Dikstra, Heresy on Mt. Athos: 
Cependant les œuvres de Boulatovitch représentent un vrai phénomène dans la culture russe : cet homme qui n'avait pas d'éducation théologique spéciale et avait mené avant la tonsure une vie éloignée de l'activité écclésiastique, ${ }^{4}$ est entré en discussion sur un problème théologique assez compliqué et s'est montré contre toute attente un meilleur connaisseur de la tradition patristique que ses adversaires, évêques et autres hommes d'Église.

C'est pourquoi les œuvres du père Antoine méritent beaucoup plus d'attention qu'on ne leur a prété jusqu'à présent. Bien que la discussion sur la vénération du nom de Dieu n'a point tiré à sa fin, les vues de Boulatovitch ne sont toujours pas devenues objet d'une étude approfondie. Aucune de ses œuvres théologiques principales n'a été traduite en une langue européenne, et seulement une d'elles a été rééditée en russe. ${ }^{5}$ Le canevas des événements historiques liés avec la querelle

Conflict over the Name of God Among Russian Monks and Hierarchs, 1912-1914 (M. Thesis. St. Vladimir' seminary, 1988), sur Internet http://www.pravoslav. de/imiaslavie/english/dykstra/dikstra.htm; Епископ Иларион (Алфевв), Священная тайна Церкви. Введение в историю и проблематику имяславских споров (Санкт-Петербург, 2002) T. I, 289-637 (l'édition française de ce livre : H. Alfeyev, Introduction à l'histoire et à la problématique des débats athonites sur la vénération du nom de Dieu (Fribourg, 2007) (Studia Ecumenica Friburgensia, 47) ; Д. АЕскин, Спор об имени Божием. Философия имени в России в контексте афонских событий 1910-х г2. (Санкт-Петербург, 2004) (Византийская библиотека) 21-137. Il faut noter que dans tous ces travaux la pensée de Boulatovitch est examinée d'une manière incomplète et assez fragmentaire ; pour les détails voir : монахиня Кассия (Т. А. СенинА), Афонское имяславие: степень изученности вопроса и перспективы исследований, Вестник Русской Христианской Гуманитарной Академии 9.1 (2008) 286-291 ; ЕАDем, Новые монографии по вопросам имяславия, Волшебная гора XV (2009) 150-166.

(4) Le père Antoine, Alexandre Ksaverievitch Boulatovitch, un ancien officier de la garde et un explorateur intrépide en Abyssinie, avant l'entrée dans les ordres menait une vie pieuse mais tout à fait éloignée de quelle que ce soit activité ecclésiastique. Sur sa vie avant la prise d'habit voir : И. КАЦНЕЛЬСОН, Г. ТереховА, По неизведанным землям Эфиопии (Москва, 1975). On peut trouver l'exposé d'un autre livre de Katsnelson consacré à Boulatovitch sur le site de Richard Seltzer: I. S. Katsnelson, A.X. Bulatovich - Hussar, Explorer, Monk (http://www.samizdat.com/kats.html).

(5) C'est Ap. Les rééditions : 1) Е. С. Полищук (ред.), Имяславие. Антология (Москва, 2002) 9-160; 2) протоиерей К. Борщ (ред., сост. и комм.), Имяславие. Сборник богословско-публициистических статей, документов и комментариев, т. I (Москва, 2003) 307-502 
onomatodoxe a déjà été assez bien étudié, mais on ne peut dire de même sur le fond théologique de la discussion.

Dans l'article présent je voudrais examiner la doctrine sur l'énergie divine formulée par le pére Antoine dans ses œuvres.

\section{Le père Antoine sur la différence entre l'énergie et l'essence en Dieu}

Le père Antoine a maintes fois souligné que la discussion sur le nom de Dieu ressemblait beaucoup au conflit entre les barlaamites et les palamites en $\mathrm{XIV}^{\mathrm{e}}$ siècle, et que cette controverse ayant commencé à cause de la prière de Jesus est devenue en fin de compte « la discussion sur l'Énergie Divine en général » $(J F, 203) .{ }^{6}$

«La thèse principale des onomatodoxes $",{ }^{7}$ affirmait le P. Antoine, « comme on le sait, est que toute énergie Divine est et se nomme Dieu, et c'est pourquoi les mots Divins inscrites dans les Saintes Écritures sont les Paroles de Dieu, pas mortes mais vivantes, et les noms de Dieu sont donc aussi en mystère Esprit et Vie, et ils peuvent avec juste raison être nommés Dieu Lui-même comme l'Énergie de la Divinité inséparable de l'Essense de Dieu » $(M P, 5)$.

Et puisque le nom de Dieu est un cas de la parole de Dieu (Ap. 17), pour démontrer sa divinité il suffit de démontrer la divinité de la vérité de la Révélation divine et en fin de compte la divinité de toute action de Dieu. La discussion sur la divinité du nom de Dieu est ramenée de cette façon au débat "sur la divinité des dons du Saint-Esprit donnés dans la prière », car les onomatoclastes contestent "le théoénergétique ${ }^{8}$ du Nom de Jesus, de tout Nom de Dieu et des Paroles de l'Evangile » et « sont arrivés à la négation de cette verité apparemment absolue que Vivante, en effet, est la parole de Dieu et efficace (Heb 4,12)» $(J F, 203)$.

C'est pourquoi dans ses œuvres le P. Antoine a assigné une place considérable aux preuves de la divinité et de la «théo-énergétique »

(6) En citant le P. Antoine je reproduis partout son emploi des lettres majuscules.

(7) J'employerai dans cet article les noms « onomatodoxes » et " onomatoclastes » employés par le P. Antoine pour appeler les partisans et les adversaires de l'onomatodoxie.

(8) Chez Bulatovitch : « Богоэнергетичность ». 


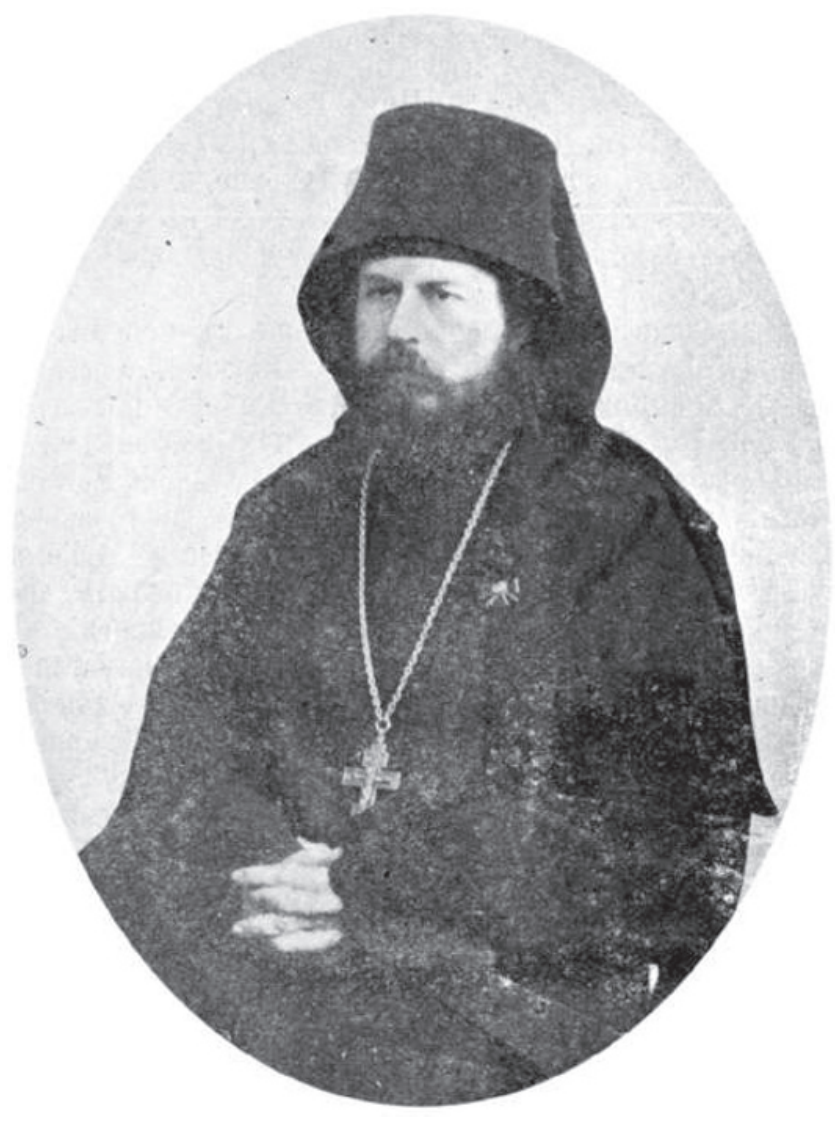

Le hiéromoine Antoine Boulatovitch 


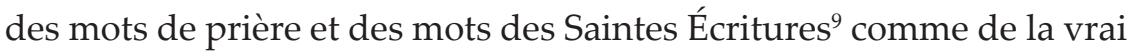
parole divine et non pas humaine, ayant accordé beaucoup d'attention au problème des énergies divines. Le débat entre les onomatodoxes et les onomatoclastes était au fond celui sur la connaissance de Dieu, sur la communion et l'union avec Dieu par les énergies divines, de la même façon qu'autrefois le débat entre les palamites et les barlaamites. La querelle onomatodoxe a commencé par la discussion sur le statut du nom de Jesus mais a révélé très vite « la différence organique de la foi » chez les onomatodoxes et les onomatoclastes sur laquelle écrivait le $P$. Antoine $(J F, 8-9)$, et cette différence concernait déjà non seulement la doctrine des noms divins mais les vues sur le salut de l'homme et sur la communion avec Dieu.

On a déjà écrit assez sur la doctrine palamite, ${ }^{10}$ et ici je voudrais montrer comment le P. Antoine s'est acquitté de sa tâche, celle de démontrer la divinité de la révélation de Dieu, « des Paroles de Dieu » au sens général, et des noms de Dieu en particulier. Ce problème n'était point simple parce que Boulatovitch n'avait pas à sa disposition une partie considérable des sources qu'employait S. Grégoire Palamas ni les œuvres de Palamas lui-même. ${ }^{11} \mathrm{Il}$ connaissait l'essentiel de la doctrine orthodoxe sur les énergies divines mais on peut dire qu'il lui fallait trouver de nouveau l'appui de cette doctrine. Le P. Antoine a su le faire quoique par d'autres moyens que S. Grégoire: Palamas en écrivant ses Triades et les autres traités citait beaucoup les œuvres dogmatiques des Pères de l'Église, mais dans les œuvres de Boulatovitch une place considérable est occupée par les citations des Saintes Écritures et des

(9) Sur cet aspect des vues de Boulatovitch voir en détail mon article: T. SEnina, The Status of Divine Revelation in the Works of Hieromonk Anthony Bulatovich, Scottish Journal of Theology (à paraître).

(10) Les œuvres principales avec une vaste bibliographie sont: J. MeyenDORFF, Introduction à l'étude de Grégoire Palamas (Paris: Édition de Seuil, 1959), citée plus bas d'après l'édition russe, И. МвйендорФ, Жизнь и труды святителя Григория Паламы. Введение в изучение. Пер. Г. Н. Начинкина под ред. И. П. Медведева и В. М. Аурье (Санкт-Петербург, 1997) (Subsidia byzantinorossica, 2); Д. И. МАКАРОв, Антропология и космология св. Григория Паламы (на примере гомилий) (Санкт-Петербург, 2003) (Библиотека христианской мысли. Исследования).

(11) La plupart des œuvres de Palamas n'étaient pas alors traduites ni même éditées. Boulatovitch, à en juger par le contenu de ses œuvres, ne connaissait que le sujet du débat entre S. Grégoire et Barlaame et l'anathème contre ceux qui nient la divinité de l'énergie de Dieu (voir plus bas la $3^{\text {ème }}$ partie de cet article), mais il ne cite pas une seule fois Palamas lui-même. 
textes liturgiques que le P. Antoine interprète dans le sens palamite. Il a consacré à cela toute " Ma pensée en Christ » au commencement de laquelle il déclarait son but :

« Je me décide à exprimer dans cet ouvrage-ci, en premier lieu, les pensées sur l'Énergie Divine qui m’ont été révélées aux moments des méditations de prière et de la lecture du Saint Evangile et des offices divins, aussi bien que les pensées qui portent un témoignage incontestable pour moi que les Paroles de la Révélation Divine adressées aux hommes sont en vérité les Paroles vivantes de Dieu Lui-même et son Énergie verbale ${ }^{12}$ et toujours existante et non pas une création de l'Énergie de la Divinité, et que les Noms de Dieu sont de pareilles Paroles Divines » $(M P, 6)$.

Les théologiens russes s'intéressaient si peu à la problématique palamite que le P. Antoine n'exagérait pas en écrivant : «La doctrine sur l'Énergie de la Sainte Trinité est élaborée très peu dans la théologie » $(M P, 6)$, en entendant la théologie russe. Peut-être le P. Antoine est devenu le premier théologien russe dont les œuvres ont mis ce sujet si fort en relief, notamment « parce que le débat présent sur le Nom de Dieu ne peut être tranché que sur la base de la doctrine sur l'Énergie Divine » $(M P, 6)$. Il donne une définition de l'énergie divine :

«L'Énergie est un terme grec, et que personne ne pense que le mot "Énergie" signifie à l'égard de Dieu la même chose que nous entendons par le mot "énergie" dans le sens commun. ... L’Énergie Divine signifie l'Activité de l’Essence Divine. ... Saint Grégoire Palamas

(12) Chez Bulatovitch : « словесная Энергия »; il entend par cela non pas littéralement l'énergie verbale dans le sens commun, mais l'énergie de Dieu qui se manifeste aux hommes par le Verbe de Dieu, par la révélation divine. Il appelle cette énergie « Глаго $九$ » (« la Parole ») en s'appuyant sur la traduction

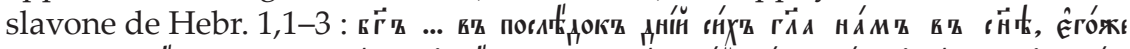

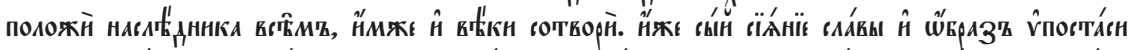

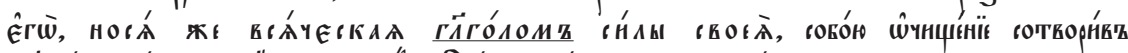

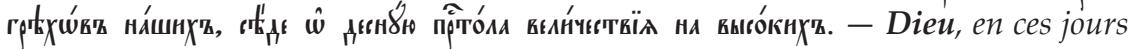
qui sont les derniers, nous a parlé par le Fils, qu'il a établi héritier de toute chose, par qui aussi il a fait les siècles. Resplendissement de sa gloire, effigie de sa substance, ce Fils qui soutient l'univers par sa parole puissante, ayant accompli la purification des péchés, s'est assis à la droite de la Majesté dans les hauteurs, - "ne comprenant pas le mot "Parole" dans le sens d'un mot articulé, mais en le comprenant dans le sens d'une Action verbale mystérieuse du Verbe ... qui définit, c'est-à-dire dit toutes les expressions de la volonté du Père », « non pas dans le sens d'un mot articulé humain, mais dans le sens spirituel et mental » $(M P, 8,20$; cf. 11). 
a établi que, d'une part, l'Activité de Dieu est sans confusion avec l'Essence de Dieu et que, d'autre part, l'Activité de Dieu est sans séparation avec l'Essence de Dieu, et il a établi la qualité Divine et la nature Divine de l'Activité de Dieu » $(M P, 6-7)$.

Ayant marqué de cette façon la succession de ses vues par rapport à la doctrine de Palamas, Boulatovitch montre comment on peut voir la différence entre l'essence et l'énergie en Dieu. L'énergie divine est communicable à la création, à la différence de l'essence non-communicable $(M P, 66)$, c'est pourquoi tous les textes d'église parlant de la communion avec Dieu, de l'action de Dieu sur l'homme, de l'acquisition de la grâce divine ont en vue l'énergie de Dieu et non pas son essence. Par exemple, «l'enrichissement en vue de Dieu » (Lk 12,21) dont parle le Christ montre, d'après l'exégèse du P. Antoine, « qu'on peut devenir possesseur de Dieu », et qu'il s'agit ici notamment de la communion à Dieu par l'énergie : "L'Essence de l'Existant n'est pas communicable à la création ... Mais l'Activité (l'Énergie) de Dieu est communicable à la création. L'Énergie Divine s'appelle Dieu et Divinité, et voici donc de quel enrichissement parle le Seigneur » $(M P, 101)$. De la même façon il interprète la phrase de la Première Épitre de Jean le Théologien "qui a le Fils a la vie » $(1 \mathrm{Jn} 5,12)$ : « Est-ce qu'il est possible d' "avoir" l'essence même du Fils de Dieu ? L'Essence de l'Existant n'est pas communicable à la création, mais à la création est communicable Son Activité (Énergie) », et par conséquent l'apôtre entendait ici notamment la communion à l'énergie divine $(M P, 162)$.

Le P. Antoine trouve les indications de la différence de l'essence et de l'énergie divines aussi dans l'hymnographie. Ainsi, par exemple, en interprétant le $2^{\text {ème }}$ tropaire de la $5^{\text {ème }}$ ode du canon à la Trinité du $4^{\text {ème }}$ ton à l'office de minuit, ${ }^{13}$ Boulatovitch écrit à propos de l'expression « la Nature incréée et la Source produisant la lumière de tout éclat qui donne la Lumière » : ici « on distingue tout à fait nettement l'Essence non-communicable de la Divinité et Son Énergie communicable » : l'essence divine est appelée « la Lumière Trisolaire, la Lumière Substantielle » etc, et l'énergie est appellée «l'Éclat qui donne la Lumière de cette Source produisant la Lumière, de l'Essence de la Sainte Trinité », et par la communion à cette énergie « on contemple la Beauté indicible de la Divinité, c'est-à-dire Ses qualités » $(M P, 34-35)$.

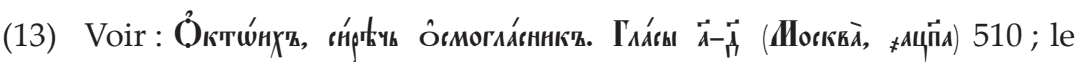

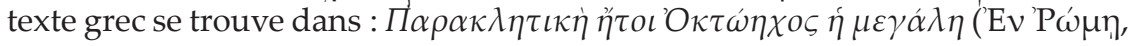
1885) 276. 
Pour appuyer cette pensée dans son autre livre le P. Antoine emploie le troisième Discours Théologique de S. Syméon le Nouveau Théologien où le saint parle de la connaissance expérimentale des actions de la Lumière divine. ${ }^{14}$ "Il est évident qu'il ne peut pas être question de la connaissance expérimentale de la Lumière des hypostases Divines et de la production de la lumière par elles ; en effet, qui peut connaître l'essence Divine "par expérience" ? ", écrit Boulatovitch en venant à la conclusion que dans le texte de S. Syméon il s'agit de la connaissance de Dieu grâce à la communion à sa lumière-énergie par la prière au nom de Dieu (JF, 35-36).

Il est intéressant de noter que pour démontrer la difference entre l'énergie et l'essence en Dieu et la possibilité de la communion à l'énergie divine, le P. Antoine utilise les œuvres de S. Syméon que les palamites n'utilisaient presque pas en leur temps, bien que dans ses œuvres le sujet de la communion à Dieu et de la divinisation soit représenté beaucoup plus fortment que chez bien d'autres Pères d'Église. La cause était qu'il y avait les différences dans la terminologie chez S. Syméon et S. Grégoire Palamas : Syméon utilisait la terminologie archaïque, confondait les notions "hypostase » et "essence " dans la christologie, enseignait la communion de Dieu «par l'essence » et $l^{\prime}$ « essentielle » connaissance de Dieu, en employant la terminologie théologique qui existait avant le système théologique de $\mathrm{S}$. Maxime le Confesseur, et la notion de l'essence chez S. Syméon différait de celle qui était généralement reçue après $S$. Maxime. En étudiant la théologie de S. Syméon Istvan Perczel a conclu que chez lui l'essence communicable « plays exactly the same role as the Operations in the Hesychast doctrine », et que les doctrines de S. Syméon et de S. Grégoire Palamas « can perhaps be considered as identical in their ultimate content, but very different in their form, and also in their philosophical connotations » $;^{15}$ cette opinion de Perczel est partagée aussi par Basile Lourié. ${ }^{16}$ On peut donc dire que les œuvres du P. Antoine représentent la

(14) Theol. III, 1. 158-168, J. Darrouzès (ed.), Syméon le Nouveau Théologien, Traités théologiques et éthiques. T. I (Paris, 1966) (SC, 122) 166; pour les detais voir : T. А. Сенина (монахиня Кассия), Св. Симеон Новый Богослов в творениях иеросхимонаха Антония (Булатовича), Волщебная гора XV (2009) 37-57, voir 45-48.

(15) I. Perczel, Saint Symeon the New Theologian and the theology of the Divine Substance, Acta Antiqua Hungarica 41 (2001) 125-146, voir 136 et 139.

(16) В. М. Аурье, Ауч света в темном веке: Симеон Новый Богослов и догматика византийских Dark Ages (à paraître). Voir aussi : Д. С. Бирюков, 
réception ecclésiastique de la doctrine de S. Syméon et en même temps correspondent aux conclusions des savants modernes : bien que Boulatovitch ne soit pas raffiné sur la doctrine du saint, il y a vu toutefois l'essentiel, à savoir que S. Syméon exprimait la même doctrine que les palamites, et que justement cette doctrine sur la communion réelle à Dieu est orthodoxe et adoptée par toute l'Église.

\section{L'énergie divine comme «l'activité triune» de la Sainte Trinité}

Le P. Antoine, en réponse à la question pourquoi l'énergie divine, malgré sa différence de l'essence, s'appelle aussi Dieu, explique :

"Ainsi que nous distinguons mais ne divisons pas les Trois Personnes de la Sainte Trinité, de même nous distinguons dans la Sainte Trinité Son Activité Triune et Son Essence, mais ne divisons point l'Activité d'avec l'Essence Divine, c'est pourquoi nous sommes en droit de nommer et nous devons nommer l'Essence Divine ainsi que l'Énergie Divine “Dieu”. Mais il est plus juste de dire “Dieu Lui-même", en entendant notamment le contact avec Dieu dans son Activité. Car en l'Activité de la Divinité on ne vénère pas une autre Divinité, séparée de la Sainte Trinité, mais la Sainte Trinité Ellemême dans la manifestation de Son Activité. Il convient donc d'appeler l'Énergie Divine manifestée aux hommes par le mot “Dieu Lui-même", pour montrer plus clairement la confession qu'elle ne se sépare pas de l'Essence Divine» $(M P, 53)$.

Cette opinion correspond parfaitement à la doctrine de S. Grégoire Palamas qui a accordé une place considérable dans ses œuvres à l'argumentation de ce pourquoi l'existence de l'énergie distincte de l'essence en Dieu ne mène pas au duothéisme : comme l'essence de Dieu est inabordable pour la création, la communion avec Dieu est possible par les énergies qui ne sont pas l'essence mais qui sont néanmoins le même Dieu, parce qu'Il « est présent tout entier dans chacune des énergies appropriées à Dieu » (Triades, III.2.7). ${ }^{17}$

Св. Симеон Новый Богослов, dans : Г. И. Беневич (сост., ред.), Д. С. БИРЮков (ред.), Антология восточно-христианской богословской мысли. Ортодоксия и гетеродоксия. Т. 2 (Москва-Санкт-Петербург: «Никея»-РХГА, 2009) (Smaragdos Philocalias ; Византийская философия, 5) 286-290.

(17) Je cite la traduction par l'édition : J. Meyendorff (éd.), Grégoire Palamas, Défense des saints hésychastes. Introduction, texte critique, traduction et notes (Louvain, 1973²) (Spicilegium Sacrum Lovaniense. Études et documents, 31) 656, avec une modification. Sur cet aspect de la doctrine de S. Grégoire, 
« Dieu est inaltérable mais aussi toujours en mouvement », écrit le P. Antoine. «Le mouvement permanent de Dieu s'exprime par la manifestation des propriétés de Son Essence » $(A p ., 4)$. Puis il interprète l'expression d'Évangile «le Verbe était avec Dieu $»^{18}$ (Jn 1,1) comme indication qu'avant la création du monde "l'action de Dieu était dirigée vers Dieu lui-même ». Dans $M P$ il revient à ce sujet et spécifie qu'en parlant dans son livre de l'énergie de Dieu, il parlera de «la manifestation extérieure de l'Activité de la Divinité par rapport à la création ", mais dans la vie intérieure de la Sainte Trinité il y a un autre mouvement : le Père engendre toujours le Fils et fait procéder l'Esprit, le Fils naît toujours et l'Esprit procède toujours du Père ( $A p ., 30$; $M P, 13)$, et outre cela il existe « le mouvement éternel des Hypostases l'une vers l'autre » (ici de nouveau Jn 1,1 est cité) qui « peut aussi être nommé l'Énergie » $(M P, 7)$. Cette énergie « intérieure » de Dieu est « la contemplation de Soi-Même », comme écrit le P. Antoine (Ap., 30), en fondant son opinion sur les paroles de $S$. Jéan Damascène que Dieu a créé le monde visible et invisible «sans se contenter de la contemplation de Lui-même $" \cdot{ }^{19}$ On trouve la même doctrine chez les autres Pères. Par example, S. Grégoire Palamas, en démontrant que les énergies divines sont incréées et éternelles, dit que la contemplation de Soi-même par Dieu est Son énergie ou activité :

«... non seulement les puissances de Dieu, que les saints Pères, comme tu pourras le trouver, appellent aussi souvent "énergies naturelles", mais aussi les œuvres de Dieu sont sans commencement ... Et la prescience divine a-t-elle un commencement? Pourrait-on imaginer un commencement de la contemplation de soi et y a-t-il eu un moment où Dieu commença à être poussé vers cette comtemplation de soi ? Jamais. ... Voici manifestement des oeuvres de Dieu sans commencement et antérieures aux siècles : la prescience,

avec les citations et les références, voir en détail : МєйендорФ, Жизнь и труды святителя Григория Паламы..., 288-300; Архиепископ Василий (Кривошеин), Аскетическое и богословское учение св. Григория Паламы, dans : Iрем, Богословские труды 1952-1983 г2. Статьи, доклады, переводы (Нижний Новгород, 1996) 145-174.

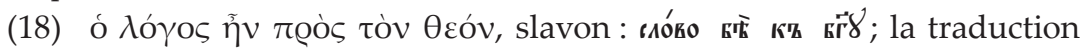
russe synodale «Слово было у Бога» est aussi possible.

(19) Cf. Joannes Damascenus, Expositio fidei, 16, 1. 2-3; voir l'édition P. B. Kotтer (hrsg.), Die Schriften des Johannes von Damaskos, vol. 2 (Berlin: De Gruyter, 1973) (PTS, 12). 
la volonté, la providence, la contemplation de soi et tout ce qui leur est semblable » (Triades, III.2.6-7). ${ }^{20}$

Le Père Antoine a réservé beaucoup de place dans $M P$ et en partie dans JF aux arguments à l'appui de la «triunité » de Dieu, c'est-à-dire que, d'une part, toutes les personnes de la Trinité possèdent l'énergie unie, mais, d'autre part, " chaque Personne prend sa propre part à l'action unie et commune ", notamment : le Père comme le Principe est « l'Initiateur c'est-à-dire Celui qui veut toute action de la Sainte Trinité », le Fils comme la Parole et l'image du Père « annonce la volonté et la décision de tout dessein de Dieu et décrit toute qualité de Dieu », et l'Esprit « est la Force vitale et le Sens ${ }^{21}$ du Père, Il est pour ainsi dire l'Âme de la Divinité », et par l'Esprit «toute volonté du Père définie par le Fils s'accomplit, et toute perfection du Père nommée par le Fils se fait sentir» $(M P, 18-19)$.

«... comme chaque Personne de la Sainte Trinité participe à toute action divine par Son Activité, nous distinguons dans l'Activité triune de la Sainte Trinité manifestée envers les hommes l'Activité particulière du Père, l'Activité particulière du Fils et l'Activité particulière de l'Esprit Saint » (MP, 13-14).

«... dans toute Action de la Sainte Trinité prennent part le Père par l'acte de volonté, le Fils par la définition et l'Esprit Saint par l'exécution et la sensation; et toute Action de la Sainte Trinité ne représente pas trois actions séparées des trois Personnes, mais elle est l'action unie du Dieu Unique » $(M P, 17)$.

«... la Faveur à l'homme et la réconciliation avec l'homme est une Action particulière du Père, la Révélation et la Vérité révélées à l'homme sont une Action particulière du Fils, et la Grâce et la Puissance données aux hommes sont une Action particulière de l'Esprit Saint, - et toutes ces trois sont une. Nous unissons ces Énergies du Père, du Fils et de l'Esprit Saint et les distiguons, toujours existantes ensemble et distinguées ; nous pensons à une seule Action inséparable de la Divinité et nous entendons les trois qui se distinguent inséparablement » $(M P, 37)$.

Chaque Hypostase de la Trinité agit, mais leur action et unie parce que « ces trois Personnes consubstantielles ont une seule essence et un seul lien de l'amour, ... une seule volonté, une seule parole, une seule sensation, un seul acte » $(M P, 18)$. Le P. Antoine a consacré à ce sujet

(20) Meyendorff, Grégoire Palamas, Défense..., 652-654.

(21) Chez Boulatovitch: « Чувство ». 
les $1^{\mathrm{er}}-3^{\mathrm{e}}$ chapitres de la première partie de $M P$ et plus tard il le touche aussi dans JF.22

Il trouve dans les Saintes Écritures des preuves que l'énergie divine est en même temps triple et une. Par exemple, les déscriptions évangéliques du baptême du Christ (Mt 3,13-17, Mk 1,9-11, Lk 3,21-22) et de Sa transfiguration (Mt 17,1-13, Mk 9,1-9, Lk 28,28-36) sont pour le P. Antoine des preuves de «la triplicité de l'activité de la Sainte Trinité » : « chaque Personne de la Sainte Trinité s'est manifestée par une action particulière de Son Activité » $(M P, 20)$. À propos des paroles du Christ « tout ce qui est à Moi est à Toi, et tout ce qui est à Toi est à Moi » $(\mathrm{Jn} 17,10)$ Boulatovitch écrit : «Le bien commun du Père et du Fils est en premier lieu l'Activité indivisible de la Divinité. Et qu'il s'agit ici notamment de l'Activité de la Divinité, c'est témoigné par les paroles précédentes : “ils ont reconnu que tout ce que Tu M'as donné vient de Toi". Par conséquent "à Moi" est tout ce que le Père donne au Fils, c'est-à-dire les Paroles et la Révélation » $(M P, 36) \cdot{ }^{23}$ Le P. Antoine trouve beaucoup de preuves de la triunité de l'énergie de Dieu dans les textes liturgiques, surtout dans les canons à la Trinité des offices de minuit de dimanche. Dans toutes les expressions comme

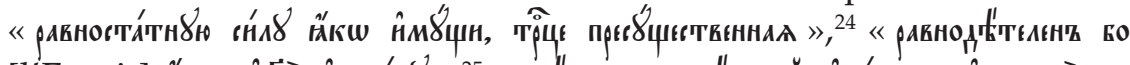

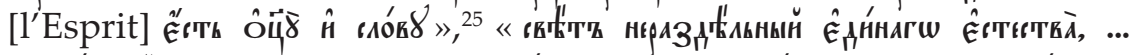

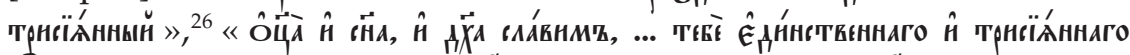

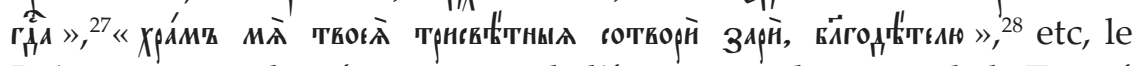
P. Antoine voit des témoignages de l'énergie triple et une de la Trinité (voir $M P, 21-36$ ).

Quel rapport cette doctrine sur l'action triune de Dieu a-t-elle avec la doctrine des Pères d'Église sur l'énergie divine? D'une part, les Pè-

(22) Voir : JF, 52-54, 92, 166, 173, 176.

(23) D'autres exemples de la même interprétation des passages différents du Nouveau Testament voir : MP, 38-39, 46-47, 49, 51-53, 84, 118-119, 221.

(24) L'office de minuit, le canon à la Trinité, ton 1 , ode 6 , tropaire 1 , en

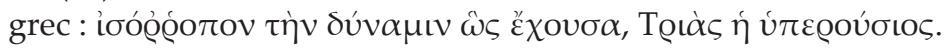

(25) Les Matines du dimanche, ton 6, la $2^{\text {ème }}$ antienne, sur la « Gloire... » :

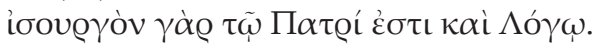

(26) L'office de minuit, le canon à la Trinité, ton 5 , ode 5 , tropaire $3: \phi \tilde{\omega} \varsigma$

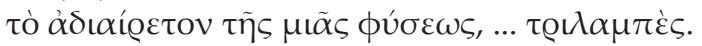

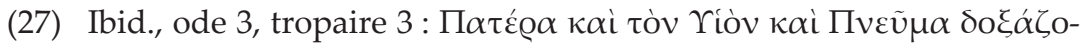

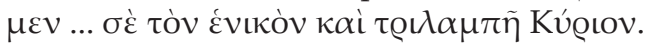

(28) L'office de minuit, le canon à la Trinité, ton 7 , ode 8 , tropaire 3 : vaóv

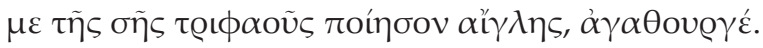


res soulignent toujours que les Hypostases de la Trinité possèdent une seule énergie parce qu'elles ont une seule essence. En résumant les arguments des Pères à l'appui de ce pourquoi l'on parle d'un seul Dieu et non pas de trois, bien qu'il y ait trois Hypostases dans la Trinité, S. Jean Damascène écrit :

« [Dans la Sainte Trinité] la communauté et l'unité se voient en réalité à cause de la co-éternité [des Personnes] et de l'identité de leurs essence, énergie et volonté, et à cause de l'unanimité dans l'opinion et de l'identité du pouvoir, de la puissance et de la bonté - j'ai dit : l'identité et non pas la ressemblance, - et aussi à cause de l'origine unie du mouvement. Car il y a une seule essence, une seule bonté, une seule puissance, une seule volonté, une seule énergie, un seul pouvoir, un seul mouvement des trois hypostases et non pas les trois pareils l'un à l'autre ${ }^{29}$

D'autre part, on peut trouver chez les Pères des affirmations fort semblables aux paroles du P. Antoine sur « l'activité particulière » des personnes de la Trinité et en même temps sur « une seule action d'un seul Dieu » :

«... Procédons donc déjà à l'exposé de la théologie, en donnant la parole au Père, au Fils et à l'Esprit Saint dont nous parlons : que l'un y mette sa complaisance, que l'autre y concoure et que l'autre l'inspire. Mieux vaut dire, que soit sur elle une seule illumination d'une seule Divinité, qui se divise en s'unissant et s'unit en se divisant, ce qui est merveilleux $» .^{30}$

"Dieu a médité premièrement les puissances des anges et des cieux. Et la pensée est devenue l'action executée par le Verbe et accomplie par l'Esprit ». ${ }^{31}$

"Cette prière ... appelle le bien de chacune des Hypostases bienheureux par un nom particulier : celui du Fils, la grâce, celui du Père, l'amour, celui de l'Esprit, la communion. Car alors que nous n'avions rien apporté de nous-mêmes et que nous étions même dignes de condamnation, le Fils est devenu Lui-même notre Saveur, parce qu'alors que nous étions encore pécheurs, Il est mort pour nous (Rom 5,8), et c'est pourquoi Sa providence de nous est la grâce. Et comme le Père s'est réconcilié avec le genre humain par les souffrances du Fils et a aimé les ennemis, Son bienfait à nous s'appelle

(29) Joannes Damascenus, Expositio fidei, 8, 1. 240-246 (КоттеR).

(30) S. Gregorius Theologus, Oraitio 28, Theologica secunda, De theologia, 1, dans : $P G$ 36, col. 25D, 28A.

(31) Idem, Oratio 45, In sanctum Pascha, 5, dans : PG 36, col. 629A. 
l'amour. Et comme il convenait aux ennemis qui s'étaient faits amis de devenir des participants des biens du Riche en miséricorde (Ephes 2,4), l'Esprit Saint tombé sur les apôtres l'accomplit, et c'est pourquoi Sa grâce envers les hommes s'appelle la communion ».32

Ainsi l'on peut dire qu'en parlant de « l'énergie triune » de Dieu, le P. Antoine, malgré l'affirmation contraire aux usages sur « l'Action particulière » de chaque Personne de la Trinité, formule la théologie qui ne sort pas du cadre de la doctrine orthodoxe dont le P. Jean Meyendorff a fait l'exposé suivant:

" ... in God's nature the decisive acting factor is hypostatic; hence, divine "energy" is not only unique but tri-hypostatic since the "energy" reflects the common life of the three Persons. The personal aspects of the divine subsistence do not disappear in the one "energy," and it is indeed the Trinitarian life of God which is communicated and participated in the "energy": through the «energy" therefore the divine hypostases appear in their co-inherence (perichōressis): "I am in the Father and the Father in me" (Jean 14:11). ... in God ... the perichōrēsis expresses the perfect love, and, therefore, the perfect unity of "energy," of the three hypostases, without however any mingling or coalescence. The "energy," because it is always Trinitarian, is always an expression and a communication of love: "As the Father has loved me, so I have loved you: abide in my love" (Jean 15:9) $» .33$

\section{3. « D'où lui vient donc tout cela ? » $(\mathrm{Mt} \mathrm{13,56)}$}

On se présente pourtant une question : d'où le P. Antoine a-t-il été renseigné sur la doctrine sur les énergies divines qui n'était point bien répandue dans la théologie russe ? En commençant le travail sur $A p$., le P. Antoine ne savait rien encore de l'existence de l'anathème contre Barlaame et il l'as appris apparemment de Michel Novoselov. ${ }^{34}$

(32) Nicolaus Cabasilas, Explicatio divinae liturgiae, 26.4, voir : R. BORNERT, J. Gouillard, P. Périchon, S. Salaville (éds.), Nicolas Cabasilas, Explication de la divine liturgie (Paris : Éditions du Cerf, 1967) (SC, 4 bis).

(33) J. MeYENDORFF, Byzantine theology: historical trends and doctrinal themes (Fordham University Press, 1979) 186. Dans le Chapitre 14 de ce livre, «The Triune God » (pp. 180-190), on trouve un exposé de la doctrine des Père byzantins sur ce problème.

(34) Sur M. A. Novoselov et son activité dans le domaine de l'instruction chrétienne voir : И. В. НикитинА, С. М. Половинкин, Московский авва, dans : Игумен Андроник ТруБачев (ред.), Архив священника Павла Александрови- 
Boulatovitch s'est adressé à Novoselov en août 1912 avec la demande d'imprimer son Ap. À ce qu'il paraît, les éditions de la «Bibliothèque religio-philosophique » publiée par Novoselov en 1902-1917 étaient connues assez bien en Athos, et le P. Antoine croyait justement que Novoselov était un homme avec des vues strictiment orthodoxes. Dans sa première lettre à Novoselov (du 13 août 1912) le P. Antoine écrit que les onomatoclastes «font descendre le Nom de Dieu sur le degré d'un simple mot, mort et créé », « nient que la puissance Divine est inhérente au Nom » et disent que le Fils de Dieu a pris le nom Jésus « seulement dans Son humanité »; que « le Nom de Dieu est devenu maintenant pour les hautains sur-savants intelligents totalement rien! Et même Dieu est devenu non pas Dieu, mais une sorte de bon camarade »; en envoyant $A p$. à Novoselov, Boulatovitch éspérait de rencontrer chez lui un écho comme chez un «intellectuel repenti ». ${ }^{35}$ Il a reçu la réponse de Novoselov très vite, car la lettre suivante du $\mathrm{P}$. Antoine à lui date du 26 septembre, et vers ce moment Boulatovitch a déjà eu le temps de remanier le début d'Ap., évidemment en rapport avec l'information reçue de Novoselov.

«Serviteur de Dieu Michel », écrit le P. Antoine, «béni soit Celui qui vous suscite à notre aide dans cette lutte. ... J'ai approuvé vos remarques. ... Je vous envoie le début remanié. Je n'ai pas eu le temps de faire tout pour ce courrier. Si les vapeurs circulent, j'enverrai le reste dans deux semaines avec le vapeur suivant. Sinon, tirez-vousen avec ce que vous avez. Il ne faut qu'abréger la Conclusion et attirer l'attention sur l'Anathème contre Barlaame $»{ }^{36}$

Un peu plus tard le P. Antoine est entré en correspondence avec le père Paul Florensky et le 17 décembre il lui apprend qu'il a renvoyé à Novoselov les six premiers chapitres d'Ap. et il écrit :

ча Флоренского. Вып. 2. Переписка с М. А. Новоселовым (Томск, 1998) 9-38 (en particulier, sur la participation de Novoselov au débat onomatodoxe voir p. 30-32). Sur le cercle de Novoselov voir : Двскин, Спор об имени Божием... 141-171. Dans sa jeunesse Novoselov s'était engoué des idées de L. N. Tolstoy mais puis il est revenu sur les positions strictement orthodoxes qu'il n'a pas quittées jusqu'à la fin de sa vie, n'étant pas pourtant clérical et ne rampant pas devant les autorités écclesiastiques.

(35) ТруБачев, Архив священника Павла Александровича Флоренского..., 70 (Lettre 25).

(36) Ibid., 72 (Lettre 26), mis en relief par moi. 
«Je vous prie d'imprimer ces mêmes chapitres et non pas ceux écrits plus tôt », dit-il, «car lorsque j'écrivais la première Apologie, je cherchais des raisons pour affirmer que l'essence du Nom est divine, mais je ne savais pas encore que la qualité Divine du Nom de Dieu est déjà reconnue par l'Eglise et que tous ce qui ne reconnaissent pas la qualité Divine de l'action du Nom de Dieu sont déjà condamnés. Par conséquent notre but est simplifié à présent, et nous avons le droit non seulement de contredire les adversaires, mais de les appeler sans façon hérétiques, et non seulement nous pouvons le faire, mais nous le devons pour sauver l'orthodoxie. ... La nouvelle Apologie est donc édifiée sur la pierre de ce que le Nom de Dieu est la Vérité révélée par Dieu, et s'il est ainsi, cette Vérité est donc l'action verbale ${ }^{37}$ de la Divinité et, par conséquent, se nomme par nécessité Dieu $» .^{38}$

Malheureusement on ne sait pas exactement ce que Novoselov a écrit à Boulatovitch, mais on peut supposer que l'association avec l'anathème contre Barlaame a pu surgir parce que les onomatodoxes insistaient dès le début que le nom divin est inséparable de Dieu, aussi bien que les palamites insistaient sur l'inséparabilité de l'énergie divine de Dieu. Dans la rédaction nouvelle d'Ap. qui a été publiée par Novoselov dans la «Bibliothèque religio-philosophique » en 1913, le P. Antoine cite l'anathème contre Barlaame et met en parallèle les débats entre les palamites et les barlaamites et les débats entre les onomatodoxes et les onomatoclastes (Ap., 2-6). Comme Boulatovitch indiquait dans $A p$., il a cité cet anathème dans sa propre traduction d'après le Triode de carême grec. ${ }^{39}$

De plus, dans la lettre à M. Novoselov du 15 mai 1913, déjà de SaintPétersbourg, le P. Antoine écrivait qu'un certain Sokolov lui avait lu un fragment inédit de $\mathrm{S}$. Grégoire Palamas où «il y a un passage disant tout franchement que le nom Dieu est appliqué en premier lieu à l'Essence divine elle-même, en deuxième lieu à chacune des Hypostases, et en troisième lieu à l'action Divine et, par conséquent, à la révélation

(37) Voir plus haut, n. 12.

(38) Трубачев, Архив священника Павла Александровича Флоренского..., 81-82 (Lettre 34), mis en relief par le P. Antoine.

(39) En la citant quelques années plus tard dans JF, Boulatovitch emploie l'édition : К. Никольский, Анафематствование (отлучение от Церкви), совершаемое в первую неделю Великого поста: Историческое исследование о чине Православия (Санкт-Петербург, 1879). 
Divine et au nom $»{ }^{40}$ Les éditeurs de cette lettre n'ont pas pu identifier ce Sokolov, mais à mon avis il y a une probabilité que il s'agit du bysantiniste russe Ivan Ivanovitch Sokolov (1865-1939), professeur de l'Académie ecclésiastique de Saint-Pétersbourg, qui étudiait les aspects historiques et théologiques de l'hésychasme byzantin et a publié en 1911 un vaste compte rendu du livre de G. Papamichaïlos sur S. Grégoire Palamas. ${ }^{41}$

Dans $A p$. le P. Antoine n'a qu'effleuré le thème de la divinité des énergies de Dieu et s'est concentré sur la divinité du nom de Dieu. Mais quand les onomatoclastes se sont mis à contester la confirmation que l'énergie divine est Dieu, ${ }^{42}$ le P. Antoine a décidé évidemment d'examiner la question des énergies divines d'une manière plus détaillée, ce qu'il a fait dans MP.

Il faut noter à part avec quelle rapidité le P. Antoine a su employer l'information reçue de Novoselov sur l'anathème contre ceux qui nient la divinité de l'énergie de Dieu, et avec quelle profondeur il a développé le thème des énergies divines dans ses œuvres, surtout dans $M P$. Ce fait paraît être étroitement lié avec celui que Boulatovitch était enraciné en pratique dans la tradition des Pères, voilà pourquoi il a su comprendre rapidement, profondément et exactement la théorie

(40) ТРубачев, Архив священника Павла Александровича Флоренского..., 101 (Lettre 49).

(41) Voir: Г. Е. ЛЕБедева, Ученый и время: И. И. Соколов, dans: И. И. Соколов, Состояние монашества в Византийской Церкви с серединь IX до начала ХІІІ века (842-1204). Опьт цеерковно-исторического исследования (Санкт-Петербург, 2003) 7-32; И. И. Соколов, Свт. Григорий Палама, архиепископ Фессалоникийский, его труды и учение об исихии. По

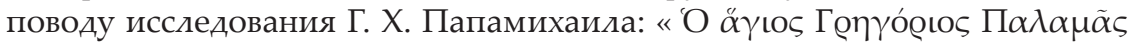

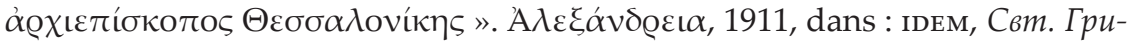
горий Палама, архиепископ Фессалоникийский, его труды и учение об исихии. Никифор Влемлид, византийский ученый и церковный деятель ХІІІ в. Церковная политика византийского илператора Исаака II Ангела (СанктПетербург, 2004) (Библиотека христианской мысли. Исследования) 46124 (réédition du compte rendu du livre de G. Papamichaïlos).

(42) La divinité de l'énergie de Dieu est niée dans l'Épître du Synode russe du 18 mai 1913 qui a condamné l'onomatodoxie comme hérésie, ainsi que dans les trois rapports sur lesquels l'Épître était fondée; voir : Cвяmoе Православие и именобожническал ересь (Харьков, 1916) [le recueil où tous les matériels onomatoclastes étaient réédités] 42-43, 48 (l'Épître), 61, 68 (rapport de l'archévêque Nicon Rozhdestvensky), 90-91 (rapport de l'archévêque Antoine Khrapovitsky), 119-122, 143-144 (rapport de Serge Troïtsky). 
et, sans connaître les œuvres de S. Grégoire Palamas et beaucoup de travaux dogmatiques des Pères byzantins examinant le problème de l'essence et des énergies divines, il pouvait formuler la théologie dans la même tradition, en trouvant avec facilité les arguments à l'appui de sa position dans d'autres sources d'Église.

\section{SUMMARY}

\section{A RUSSIAN PALAMITE IN THE EARLY TWENTIETH CENTURY: HIEROMONK ANTHONY BULATOVITCH AND HIS TEACHING ON THE DIVINE ENERGY}

One side of the theological thought of hieroschemamonk Anthony (Bulatovitch, +1919) is reviewed, namely, his teaching on the divine energy. Fr Anthony was the leader of the Athonite onomatodoxes in the 1910s; his works remain so far mostly unstudied. The teaching of the onomatodoxes on the name of God as a divine revelation of God and the divine energy was going back to the teaching of St Gregory Palamas on the divine energy of God. Thus, Fr Anthony develops, taking evidence from the Holy Scripture and the hymnography, a doctrine on the tri-unity of the energy of the Holy Trinity as the unique energy of the three Persons and the unique God. This doctrine remains strictly within the frame of the eastern patristic tradition, which was, however, almost forgotten in the official ecclesiastical education and textbooks of the contemporary Russian Church. 